\title{
Suicide par injection de T 61
}

\section{Suicide by injection of T-61}

\section{Françoise ANGER $^{(1)}$, François PAYSANT ${ }^{(2)}$, Alain BAERT ${ }^{(2)}$, François LE GALL ${ }^{(2)}$, M. A. LE GUEUT ${ }^{(2)}$, Jean-Pierre GOULLÉ ${ }^{(3)}$, Gérard LACHÂTRE ${ }^{(4)}$, Gilbert PÉPIN(5), Jean-Pierre ANGER*(1)}

(1) Laboratoire de Toxicologie, Faculté de Pharmacie; Université de RENNES 1

(2) Laboratoire de Médecine Légale, Faculté de Médecine, Université de RENNES 1 (3) Laboratoire de Pharmacocinétique et de Toxicologie Clinique, Groupe Hospitalier - 76083 LE HAVRE Cedex

(4) Service de Pharmacologie et Toxicologie, CHU Dupuytren - 87042 LIMOGES

(5) Laboratoire d'Expertises TOXLAB, 7, rue Jacques Cartier - 75018 PARIS

* Auteur à qui adresser la correspondance : Jean-Pierre ANGER, Laboratoire de Toxicologie, Faculté de Pharmacie, 2, Avenue du Professeur Léon Bernard - 35043 RENNES Cedex Tél : 0223234867 - Fax : 0223234794

(Reçu le 2 avril 2002 ; accepté le 15 mai 2002)

\section{$R \hat{E} S U M E$}

Le T-61 ou TANAX® est un agent euthanasiant vétérinaire parfois impliqué dans les tentatives de suicide chez l'homme en administration par différentes voies. Le produit commercial est un mélange de trois principes actifs : l'embutramide, le mébezonium et le chlorhydrate de tétracaïne dont l'association entraîne plus ou moins rapidement la mort par anoxie cérébrale. Nous rapportons ici le cas d'une jeune femme décédée des suites de l'injection intraveineuse de T 61. À ce propos nous faisons le point sur les données de la littérature concernant les modes d'intoxication par le T-61, les possibilités analytiques de mise en évidence et de dosage de l'embutramide ainsi que les rares données humaines ex expérimentales relatives aux concentrations retrouvées dans les milieux biologiques en cas de suicide ou d'euthanasie animale.

\section{MOTS-CLÉS}

$T$ 61, Embutramide, Intoxication suicide, Dosage, Milieux biologiques.

\section{SUMMARY}

T-61 or TANAX®, a commonly used euthanasic agent in veterinary medicine, is sometimes used by humans in suicide attempts. It can be administred by different routes. The commercial product consists of a mixture of three active compounds : embutramide, mebezonium and tetracaine hydrochloride. Their association causes brain anoxy and death. We report here the case of a young woman who died after an intravenous injection of T-61. On this subject we reviewed the existing data concerning the routes of intoxication and the analytical methods to detect and quantify embutramide as well as the rare human and experimental data about embutramide concentrations found in biological materials in cases of suicide or animal euthanasia.

\section{KEY-WORDS}

$T$ 61, Embutramide, Suicide, Determination, Biological materials. 


\section{Introduction}

Le T 61 (TANAX®) est un agent euthanasiant vétérinaire commercialisé par le Laboratoire INTERVET, B. P. 17144 , 49071 BEAUCOUZE Cedex. Il se présente en flacon de $50 \mathrm{ml}$ de solution injectable associant trois principes actifs :

- l'embutramide $(200 \mathrm{mg} / \mathrm{ml})$ ou $\mathrm{N}$-2-éthyl-2-(3méthoxyphényl)-butyl-4-hydroxybutanamide ;

- l'iodure de mébézonium $(50 \mathrm{mg} / \mathrm{ml})$ ou di iodure de 4,4'-méthylène bis- $\mathrm{N}, \mathrm{N}, \mathrm{N}$-triméthylcyclohexanaminium ;

- le chlorhydrate de tétracaïne $(5 \mathrm{mg} / \mathrm{ml})$ ou chlorure de p-butylaminobenzoyl-diméthylamino-éthanol.

Ces trois composés sont dissous dans un mélange de $\mathrm{N}$, $\mathrm{N}$-diméthylformamide (DMF) et d'eau distillée $(6: 4$, $\mathrm{v} / \mathrm{v})$.

Le produit commercial est habituellement utilisé pour l'euthanasie des bovins, ovins, caprins, équins, porcins, volailles, lapins, chiens et chats. Il est administré par voie intraveineuse ou intracardiaque à raison de $0,3 \mathrm{ml} / \mathrm{kg}$ de poids ou éventuellement par voie intrapulmonaire. Chez l'animal l'action conjuguée des trois principes actifs produit l'effet maximal létal recherché : la mort survient en quelques instants par anoxie cérébrale.

L'embutramide est un anesthésique général dérivé du gamma-OH qui possède un effet narcotique puissant et induit une anesthésie profonde en paralysant les centres cérébraux qui contrôlent la respiration dans le système nerveux central.

L'iodure de mébézonium exerce une action curarimimétique paralysant les muscles squelettiques et induisant rapidement un collapsus respiratoire dû à une paralysie des muscles intercostaux et du diaphragme.

Le chlorhydrate de tétracaïne appartient au groupe des anesthésiques locaux comme la procaïne mais est vingt fois plus toxique. Il est ajouté à la solution en raison de son action anesthésique locale qui réduit la réaction douloureuse tissulaire au point d'injection.

Le DMF est un solvant organique d'utilisation très répandue notamment dans l'industrie des plastiques.

Chez l'homme, les observations d'intoxications accidentelles au T-61 rapportées concernent des travailleurs exposés et sont toujours le fait d'une contamination cutanée et/ou pulmonaire. Ce produit est parfois utilisé à des fins suicidaires le plus souvent par injection intraveineuse ou intracardiaque, plus rarement par ingestion. C'est le fait des vétérinaires ou de leur entourage, parfois des médecins (1). La zone d'injection est généralement le poignet ou l'avant-bras. Le décès peut être immédiat ou survient de façon retardée.
Nous rapportons ici le cas d'une jeune femme décédée des suites d'un syndrome asphyxique majeur après injection I.V volontaire de T 61 .

\section{Circonstances médico-légales}

Le 29 Décembre 2000, une jeune femme de vingt ans est découverte décédée allongée sur son lit dans un foyer de jeunes travailleurs. Un dispositif de perfusion est en place, l'aiguille installée au pli du coude. Le produit de la perfusion est vite identifié. Il est identique à celui d'un flacon retrouvé à proximité du corps : il s'agit de $\mathrm{T} 61$, un produit euthanasiant vétérinaire. Selon les données de l'enquête, cette jeune femme exerçait depuis peu de temps les fonctions d'aide vétérinaire dans une clinique où elle avait pu avoir accès à l'armoire aux produits dangereux. Le produit de la perfusion aurait donc été subtilisé sur son lieu de travail. L'enquête a également montré qu'il s'agissait d'une jeune femme dépressive connue pour avoir déjà tenté plusieurs fois de se suicider.

\section{Constatations autopsiques}

À l'examen externe du corps, il est noté une cyanose majeure avec hémorragies sous conjonctivales bilatérales, pétéchies diffuses de la partie supérieure du thorax et de l'extrémité céphalique. Il est par ailleurs noté un aspect de brûlure de la face interne du bras sur le trajet de la veine perfusée. À l'ouverture du corps, les poumons, le cerveau et les viscères de la cavité abdominale (foie et reins) sont congestifs.

Un prélèvement de sang a été pratiqué dans la veine sous clavière gauche. Ce prélèvement ainsi qu'un fragment de foie et un fragment de cerveau ont fait l'objet d'une analyse toxicologique.

\section{Constatations histologiques}

L'examen anatomo-pathologique a porté sur les poumons et un fragment de foie. Après coloration des coupes tissulaires à l'hémalun-éosine-safran, les observations suivantes ont été formulées :

Au niveau du poumon, l'axe broncho vasculaire apparaît libre de thrombose. Le revêtement bronchique est conservé de même que les structures cartilagineuses et glandulaires. On constate simplement une dilatation des capillaires de la paroi. Les ganglions satellites sont le siège d'une dilatation sinusale. Le parenchyme pulmonaire, au niveau des trois lobes, est le siège de quelques lésions de pseudo emphysème. On note surtout des zones d'alvéolite œdémateuse et hémorragique 
avec présence de nombreux sidérophages. Les septa alvéolaires apparaissent très congestifs. La plèvre n'est pas le siège de modifications lésionnelles, mais en certaines zones, on constate une majoration des lésions de pseudo emphysème en zone sous pleurale. Au niveau du lobe inférieur, ces lésions apparaissent plus accentuées, sous forme d'un œdème plus massif, accompagnées d'une turgescence vasculaire et parfois destruction des septa alvéolaires.

Au niveau hépatique, on observe d'emblée une dilatation des veines centro lobulaires, accompagnée d'une dilatation sinusale importante. Quelques espaces portes apparaissent très modérément inflammatoires. Il n'a pas été constaté de zone de nécrose.

En conclusion, l'examen anatomo-pathologique révèle l'existence de lésions pulmonaires et hépatiques de type aigu. Ces aspects lésionnels, sans signe de chronicité, sont le reflet d'une insuffisance cardiaque brutale.

\section{Matériel et méthodes}

\section{Recherche toxicologiques générales dans le sang}

Les molécules volatiles (alcools, acétone, divers solvants) sont recherchées par chromatographie en phase gazeuse selon la technique de l'espace de tête (GC-HS) sur un analyseur GENESIS VARIAN (Les Ulis, France) couplé à un chromatographe en phase gazeuse VARIAN 3400 (2). Cet appareil, équipé d'un détecteur à ionisation de flamme, est muni d'une colonne DBWAX de marque J \& W SCIENTIFIC dont la longueur est 30 mètres et le diamètre $0,53 \mathrm{~mm}$ (épaisseur du film 1,0 micron). La recherche d'oxyde de carbone est réalisée sur le sang total fluoré par une méthode spectrophotométrique, à l'aide d'un CO-OXYMETRE de marque Instrumentation Laboratory, modèle IL-242 (Paris, France).

Les dépistages ou dosages immunologiques des principaux médicaments responsables d'intoxication (barbituriques, benzodiazépines, antidépresseurs tricycliques, amphétamines, cocaïne, opiacés, digoxine, digitoxine, methadone, paracétamol, propoxyphène, salicylés) sont effectués par immuno-polarisation de fluorescence FPIA sur automates TDX et AXSYM Abbott (Rungis, France), à l'aide des réactifs destinés à ces appareils. Une recherche toxicologique complémentaire par chromatographie en phase gazeuse et spectrométrie de masse (GC-MS) est réalisée sur l'échantillon biologique en vue de rechercher les substances les plus souvent retrouvées lors des tentatives d'autolyse : médicaments, stupéfiants, pesticides et autres produits toxiques. À $1,5 \mathrm{ml}$ de sang, on ajoute
$3,5 \mathrm{ml}$ d'eau. Après extraction réalisée par Toxitube $\circledast$ A (Amilabo, Chassieu, France), la phase organique évaporée est reprise par $100 \mu \mathrm{l}$ de méthanol dont $1 \mu \mathrm{l}$ est injecté sur une colonne capillaire de HP-5-MS et séparation sur un chromatographe en phase gazeuse HP 6890 couplé à un détecteur de masse 5973.

\section{Dosage de l'embutramide dans le sang et les viscères}

\section{Réactifs}

Les solvants sont de qualité chromatographie liquide haute performance (CLHP), les autres produits chimiques sont de qualité pour analyse (Prolabo, Chelles, France). L'embutramide nous a été fourni gracieusement par le Docteur Y. GAILLARD (Toxlab, Paris, France) ; il permet de préparer une solution mère à $1 \mathrm{~g} / 1$ ainsi que trois solutions filles à 1,10 et $100 \mathrm{mg} / \mathrm{l}$ pour la gamme étalon L'étalon interne est la codéine $d_{3}$ Radian (Promochem, Molsheim, France). Le réactif de dérivation est du bis-silyl-trifluoro-acétamide (BSTFA) avec $1 \%$ de triméthylchlorosilane (TMCS) (Interchim, Montluçon, France).

\section{Dosage de l'embutramide dans le sang}

Le dosage de l'embutramide dans le sang est effectué sur Toxitube ${ }^{\circledR} A$ après extraction d'une dilution du sang auquel on ajoute la codéine $d_{3}$ (étalon interne, $E I$ ). Après évaporation, l'extrait obtenu est dérivé par le BSTFA $+1 \%$ de TMCS. Une gamme étalon contenant 1,10 et $100 \mathrm{mg} / \mathrm{l}$ est traîtée dans les mêmes conditions. L'acquisition est réalisée en mode impact électronique de 40 à 500 uma avec une programmation de température de $40^{\circ} \mathrm{C}$ à $300^{\circ} \mathrm{C}$, après injection de $1 \mu \mathrm{l}$ de l'extrait dilué. La quantification fait appel à l'extraction des ions majoritaires : 190 pour l'embutramide et 374 pour la codéine $\mathrm{d}_{3}(\mathrm{EI})$

\section{Dosage de l'embutramide dans les viscères}

Les viscères (foie et cerveau) sont préparés par broyage dans un appareil Retsch (Bioblock, Illkirch, France). Le dosage de l'embutramide est effectué en appliquant la méthode recommandée par la Société Française de Toxicologie Analytique (SFTA) pour le dosage des morphiniques et des cocaïniques dans le sang (3). Il s'agit d'une triple extraction en phase liquide (heptane, chloroforme, isopropanol) en présence de codéine $d_{3}$ utilisée comme étalon interne, suivie d'une silylation. $540 \mathrm{mg}$ de cerveau et $510 \mathrm{mg}$ de foie sont broyés dans $5 \mathrm{ml}$ de tampon d'extraction à $\mathrm{pH} 8,4$. Les dosages sont réalisés en double en prélevant 1 et $2 \mathrm{ml}$ de la solution obtenue pour chaque viscère. Une gamme étalon préparée à partir des solutions filles est traitée dans les mêmes conditions. L'acquisition est réalisée en mode impact électronique de 40 à 500 uma avec une programmation de température de $40^{\circ} \mathrm{C}$ à $300^{\circ} \mathrm{C}$ après 
injection de $1 \mu \mathrm{l}$ de l'extrait dérivé. La quantification fait appel à l'extraction des ions majoritaires : 190 pour l'embutramide et 374 pour la codéine $d_{3}$ (EI). Les conditions chromatographiques sont identiques à celles utilisées pour le dosage sanguin.

\section{Résultats}

\section{Recherches toxicologiques générales dans le sang}

Les recherches d'alcools et des molécules volatiles autres sont négatives. On note l'absence d'oxyde de carbone. Les dépistages ou dosages immunologiques sont négatifs.

L'analyse par GC-MS révèle la présence d'un pic très important d'embutramide (figure 1), identifié par son spectre de masse (figure 2) à l'exclusion de tout autre xénobiotique détectable.

\section{Dosage de l'embutramide dans le sang et les viscères}

Les résultats sont rapportés dans le Tableau I

Tableau I : Concentrations en embutramide retrouvées dans les prélèvements biologiques examinés.

\begin{tabular}{|l|c|c|c|}
\hline Milieu biologique & $\begin{array}{c}\text { Sang total } \\
\end{array}$ & $\begin{array}{c}\text { Foie } \\
(\mathbf{m g})\end{array}$ & $\begin{array}{c}\text { Cerveau } \\
(\mathbf{m g} / \mathbf{k})\end{array}$ \\
\hline Embutramide & 751 & 64,4 & 14 \\
\hline
\end{tabular}

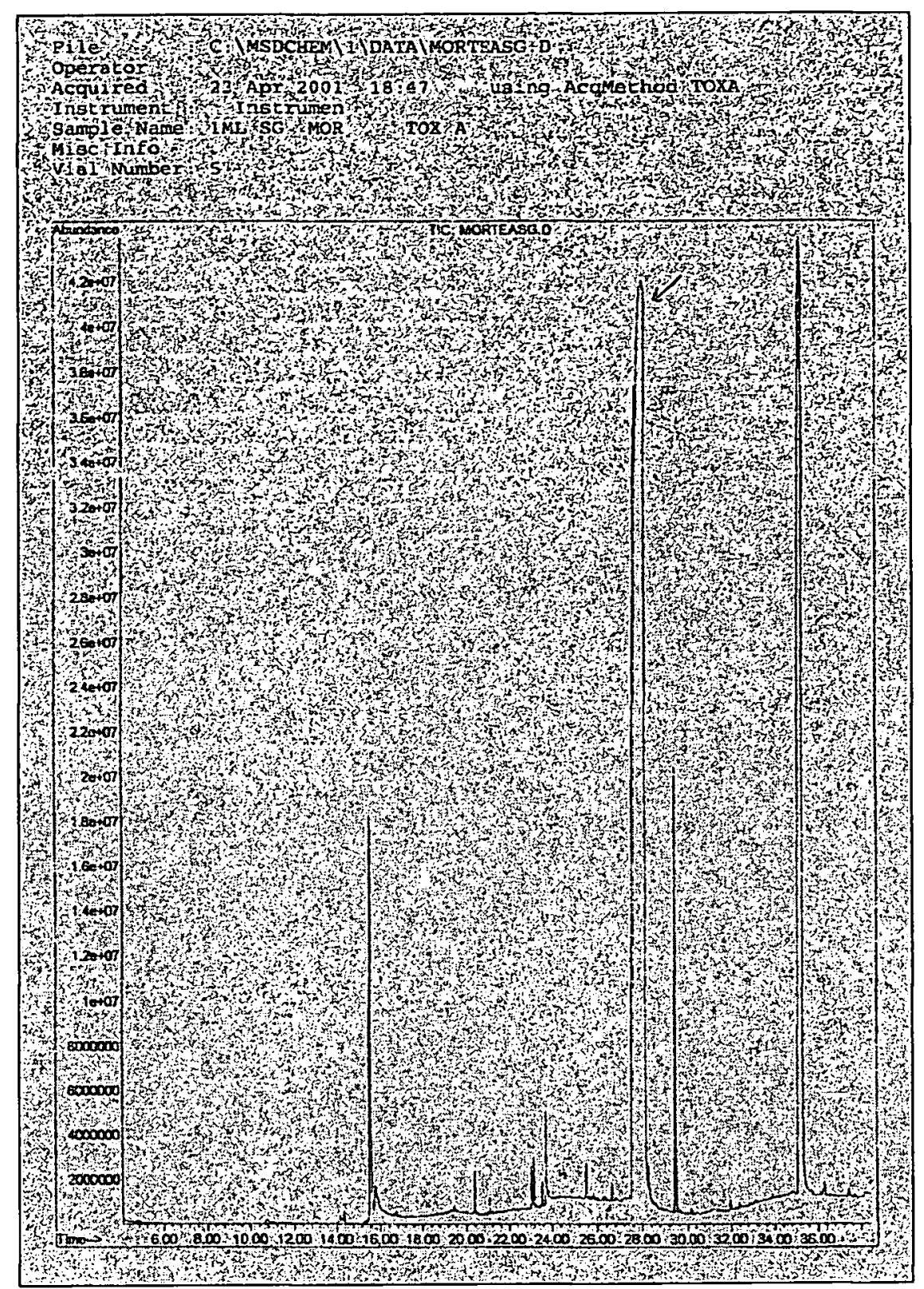

Figure 1 : Chromatogramme (GC-MS) de l'extrait sanguin. $N B$ : La flèche indique le pic de l'embutramide.

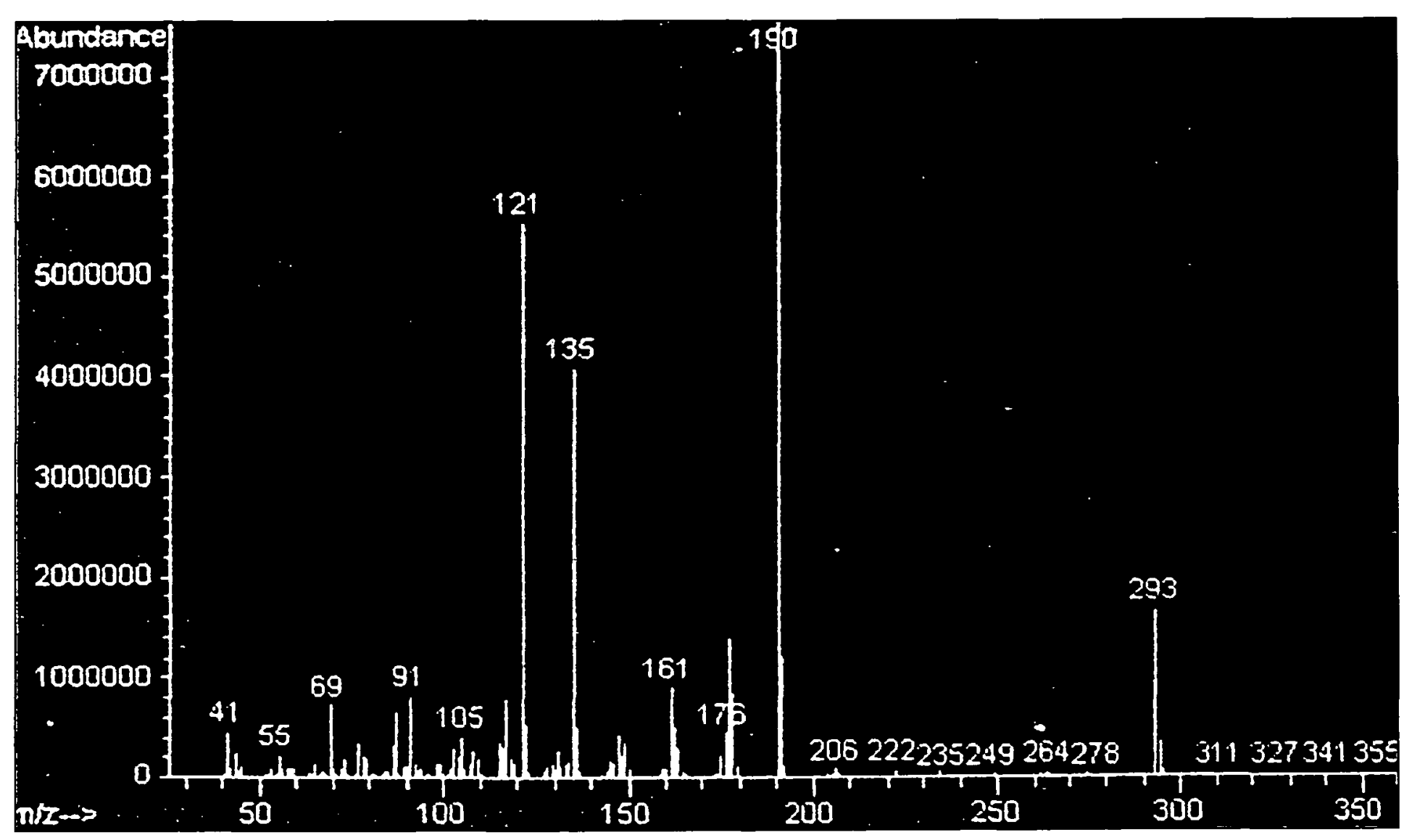

Figure 2 : Spectre de masse de l'embutramide. 


\section{Commentaires}

Les autres constituants du T 61 , iodure de mébézonium, chlorhydrate de tétracaïne et diméthylformamide n'ont pu être identifiés, ni quantifiés. En ce qui concerne l'iodure de mébézonium, ce curare pose des problèmes d'extraction. Quant à la tétracaïne, le spectre de masse sanguin relativement pauvre pour cette méthode, est constitué essentiellement d'ions de petite masse (58 et 71) qui n'ont pas permis de l'identifier formellement. Le dosage de l'embutramide a été tenté par CLHP, mais cette technique a dû être abandonnée en raison d'un manque de reproductibilité.

\section{Discussion}

Le T 61 peut faire l'objet d'intoxications accidentelles par voie sous cutanée ou projection oculaire mais le plus souvent il s'agit d'intoxications volontaires par injection sous cutanée, intraveineuse ou par ingestion. Les quantités administrées lors des tentatives d'autolyse vont de 5 à $40 \mathrm{ml}$ pour les voies parentérales et généralement la totalité du flacon commercial $(50 \mathrm{ml})$ pour la voie orale $(1,4,5,6,7)$. L'évolution de l'intoxication dépend évidemment des modalités de l'administration du produit, de la quantité administrée et d'une éventuelle assistance médicale. L'injection intraveineuse reste certainement la plus dangereuse : une dose de $40 \mathrm{ml}$ de $\mathrm{T} 61$ induit chez l'homme un coma rapide et la mort quasi instantanée. L'injection sous cutanée est moins dangereuse et peut éventuellement occasionner une réaction inflammatoire qui disparaît après traitement (8). Du fait de sa simplicité, l'ingestion orale est la voie d'intoxication la plus fréquente lors des tentatives de suicide et dans de nombreux cas ces intoxications ont pu évoluer favorablement à condition de les traiter rapidement mais il s'y associe généralement une hépatotoxicité tardive vraisemblablement due au DMF présent dans la préparation et qui est métabolisé au niveau hépatique en méthylisocyanate induisant une nécrose cellulaire (6).

D'un point de vue analytique, l'identification et le dosage des constituants du T 61 dans les milieux biologiques humains ou animaux permettent de confirmer le diagnostic de l'intoxication. Depuis 1983 plusieurs méthodes analytiques ont été proposées pour détecter les composants du T 61, même si quelques difficultés subsistent. Il n'est pas aisé, en effet, de caractériser l'iodure de mébezonium à partir des milieux biologiques du fait de la très faible solubilité des composés ammoniums quaternaires dans la plupart des solvants organiques. Signalons cependant que la mise en évidence de l'iode par ICP-MS dans le sang d'un chien euthanasié par le T 61 s'est révélée significativement supérieure à la normale (G. Pepin, communication personnelle). Par ailleurs le chlorhydrate de tétracaïne est rapidement hydrolysé par les enzymes estérasiques. L'embutramide reste donc le seul constituant de cette formulation, le plus fréquemment recherché.

En 1983, Bertol et al (9) décrivent deux méthodes analytiques différentes pour détecter l'embutramide dans le sang et l'urine. Après extraction du milieu biologique alcalinisé par un solvant organique et chromatographie sur couche mince de l'extrait, le spot obtenu est elué et dosé par spectrophotométrie ultraviolette. Pour confirmer une intoxication humaine ou animale au $\mathrm{T}$ 61, Braselton et al (10) en 1989 développent un procédé permettant d'identifier l'embutramide dans les tissus et les milieux biologiques par GC-MS. La quantification est obtenue selon le mode SIM des masses $\mathrm{m} / \mathrm{z}$ 135, 190 et 293. À l'occasion d'une intoxication suicidaire, Smith et Lewis (4) en 1989 réussissent à identifier en plus de l'embutramide, la tétracaïne par une méthode par GC-MS dans un extrait organique du contenu stomacal de la victime. Les auteurs reconnaissent n'avoir pas pu extraire l'iodure de mébezonium par ce procédé, mais ils détectent sur le chromatogramme de l'iodure de méthyle, probablement par suite de la pyrolyse du groupe triméthylamino de la molécule. Une autre méthode pour le dosage de l'embutramide est proposée par Huo et al, (11) en 1994 où l'embutramide est détecté dans les matrices biologiques par CPG équipée d'un détecteur azote phosphore. En 1997, Gaillard et Pépin (12) décrivent une méthode générale d'identification de 600 substances d'intérêt toxicologique dont l'embutramide, par HPLC couplée à un détecteur à barrettes de diodes. En 1998, Hooijerink et al, (13) développent une méthode d'extraction de l'embutramide à partir des tissus biologiques sur colonne Extrelut ${ }^{\circledR}$ ou en phase solide C 18 , suivie d'une élution par le méthanol. Les phases organiques sont évaporées sous courant d'azote et les résidus silylés par un mélange MSTFA-TMCS. Le dosage est réalisé par GC-MS selon le mode impact electronique ou par ionisation chimique. La limite de quantification se situe entre 50 et $100 \mu \mathrm{g} / \mathrm{kg}$. Les dernières techniques en date sont dues à Giorgi et al, (14) en 2001 qui utilisent et comparent l'HPLC et la GC-MS. Le sang tamponné à $\mathrm{pH}$ 8,5-9 est extrait par le dichlorométhane. La séparation de l'embutramide par HPLC est réalisée sur une colonne ODS2 $\mathrm{C} 185 \mu$ en présence d'une phase mobile $\mathrm{CH}_{3} \mathrm{OH}-\mathrm{H}_{2} \mathrm{O}(65: 35)$ ajustée à $\mathrm{pH} 4,7$ avec $\mathrm{H}_{3} \mathrm{PO}_{4}$ et détection par spectrophotométrie UV à $273 \mathrm{~nm}$. La limite de détection est de $2,5 \mu \mathrm{g} / \mathrm{ml}$. La séparation et l'identification de l'embutramide par GC sont réalisées sur colonne capillaire OV1 avec programmation de température et détection par spectrométrie de masse en mode SIM et acquistion sur les ions $\mathrm{m} / \mathrm{z}: 121,135$ et 
190. La limite de détection est de $250 \mathrm{ng} / \mathrm{ml}$.

La technique GC-MS que nous avons utilisée est tout à fait comparable à celles qui sont décrites récemment dans la littérature. Le taux d'embutramide retrouvé dans le sang de notre victime est certes très important, mais la dose qu'elle s'est administrée a été estimée à environ $200 \mathrm{mg} / \mathrm{kg}$ et la valeur trouvée est approximativement la moitié de celle observée expérimentalement chez le rat euthanasié à la dose de $400 \mathrm{mg} / \mathrm{kg}$ par cette même voie. La concentration annoncée pour le foie est également en accord avec les données de la littérature.

Nous avons rassemblé dans le tableau II les concentrations en embutramide retrouvées dans le sang, le foie et le cerveau chez l'homme après intoxication volontaire par différentes voies ou chez l'animal après euthanasie, le plus souvent par voie intraveineuse. Comme on pourra le constater, les données humaines sont rares et les doses administrées sont souvent mal connues ce qui complique encore plus l'interprétation des résultats.

\section{Conclusion}

À l'occasion d'une intoxication humaine dans un but suicidaire par injection intraveineuse de T-61, un agent euthanasiant utilisé en médecine vétérinaire, nous rapportons les constatations autopsiques, anatomo-pathologiques et toxicologiques effectuées sur la victime. Seul l'un des constituants de la préparation commerciale, l'embutramide a pu être identifié et quantifié dans le sang, le foie et le cerveau. Nous en profitons pour rappeler les différents types d'intoxication humaine avec le T-61 ainsi que les techniques analytiques récentes permettant la mise en évidence et le dosage de l'embutramide dans les milieux biologiques.

Remerciements : Nous remercions le Docteur Vétérinaire J-M HELIEZ de nous avoir aimablement fourni un échantillon de T-61 pour la mise au point de nos déterminations analytiques.

Tableau II : Concentrations sanguine et tissulaire de l'embutramide retrouvées lors de suicides ou chez des animaux après euthanasie au T-61.

\begin{tabular}{|c|c|c|c|c|c|c|c|}
\hline Victime & Sexe & \begin{tabular}{|l|} 
Voie \\
d'administration
\end{tabular} & Dose & Sang & Foie & Cerveau & Références \\
\hline M... S & $\mathrm{F}$ & IV & $\approx 200 \mathrm{mg} / \mathrm{kg}$ & $751 \mathrm{mg} / \mathrm{l}$ & $64,4 \mathrm{mg} / \mathrm{kg}$ & $14 \mathrm{mg} / \mathrm{kg}$ & Notre résultat \\
\hline $\begin{array}{l}\text { M... F } \\
\text { P... Al } \\
\text { C... A }\end{array}$ & $\begin{array}{l}\mathrm{M} \\
\mathrm{F} \\
\mathrm{H}\end{array}$ & $\begin{array}{l}\text { IV } \\
\text { Orale } \\
? \\
\text { IV } \\
? \\
\text { intra-pulmonaire }\end{array}$ & $\begin{array}{l}150 \mathrm{mg} / \mathrm{kg} \\
? \\
55 \mathrm{mg} / \mathrm{kg}\end{array}$ & $\begin{array}{l}33,7 \mathrm{mg} / 1 \\
15,3 \mathrm{mg} / 1 \\
226 \mathrm{mg} / 1 \\
23,9 \mathrm{mg} / 1 \\
43 \mathrm{mg} / 1\end{array}$ & $10,5 \mathrm{mg} / \mathrm{kg}$ & & $\begin{array}{l}\text { G.Pépin } \\
\text { (communication personnelle) } \\
\text { G. Lachâtre } \\
\text { (communication personnelle) } \\
\text { P. Kintz } \\
\text { (communication personnelle }\end{array}$ \\
\hline & $\mathrm{F}$ & Orale & $\approx 300 \mathrm{mg} / \mathrm{kg}$ & $31 \mathrm{mg} / \mathrm{l}$ & $12 \mathrm{mg} / \mathrm{kg}$ & & $\begin{array}{l}\text { Smith R.A., Lewis D } \\
\text { Vet Hum Toxicol } \\
1989,31(4): 314-320\end{array}$ \\
\hline $\begin{array}{l}\text { Chien } \\
\text { Chien } \\
\text { Mouton } \\
\text { Porc } \\
\text { Bœuf } \\
\text { Rat } \\
\text { Cheval } \\
\text { Porc }\end{array}$ & & $\begin{array}{l}\text { IV } \\
\text { IV } \\
? \\
? \\
? \\
\text { IV } \\
\text { IV } \\
\text { IV }\end{array}$ & $\begin{array}{l}62 \mathrm{mg} / \mathrm{kg} \\
66 \mathrm{mg} / \mathrm{kg} \\
48 \mathrm{mg} / \mathrm{kg} \\
52 \mathrm{mg} / \mathrm{kg} \\
78 \mathrm{mg} / \mathrm{kg} \\
400 \mathrm{mg} / \mathrm{kg} \\
20 \mathrm{mg} / \mathrm{kg} \\
100 \mathrm{mg} / \mathrm{kg}\end{array}$ & \begin{tabular}{|l|}
$250 \mathrm{mg} / \mathrm{l}$ \\
$340 \mathrm{mg} / \mathrm{l}$ \\
$61 \mathrm{mg} / \mathrm{l}$ \\
$12 \mathrm{mg} / \mathrm{l}$ \\
$18 \mathrm{mg} / \mathrm{l}$ \\
$1352 \pm 407 \mathrm{mg} / \mathrm{l}$ \\
$17,6 \pm 9,3 \mathrm{mg} / \mathrm{l}$ \\
$814 \pm 372 \mathrm{mg} / \mathrm{l}$
\end{tabular} & $\begin{array}{l}15 \mathrm{mg} / \mathrm{kg} \\
30 \mathrm{mg} / \mathrm{kg} \\
8,9 \mathrm{mg} / \mathrm{kg} \\
5,4 \mathrm{mg} / \mathrm{kg} \\
\\
146 \pm 94 \mathrm{mg} / \mathrm{kg} \\
17,8 \pm 7,5 \mathrm{mg} / \mathrm{kg}\end{array}$ & $\begin{array}{l}180 \mathrm{mg} / \mathrm{kg} \\
210 \mathrm{mg} / \mathrm{kg} \\
85 \mathrm{mg} / \mathrm{kg} \\
14 \mathrm{mg} / \mathrm{kg} \\
6,3 \mathrm{mg} / \mathrm{kg}\end{array}$ & $\begin{array}{l}\text { Braselton W.E. } \\
\text { Ray J-S } \\
\text { Vet. Hum Toxicol } \\
\text { 1988, } 30(6): 536-539 \\
\text { Giorgi et al } \\
\text { J.Anal. Tox., } \\
2001,25: 323-27\end{array}$ \\
\hline
\end{tabular}




\section{Références}

1. Meram D., Chabac S., Rouzioux J-M., Descotes J. Intoxications aiguës par le T 61. Bilan des Centres AntiPoisons. J. Toxicol. Clin. Exp., 1989, 9, 5 : 340-42.

2. Goullé J.P., Lacroix C., Anagnostides J., Gharios G., Perdue-Legendre E., Rigault J.P., Nouveau J. L'acétone : un marqueur biologique trop souvent négligé. Toxicorama, 1999, $11: 265-269$.

3. Gaillard Y., Pépin G., Marquet P., Kintz P., Deveaux M., Mura P. Identification et dosage de la benzoylecgonine, cocaïne, méthylecgonine ester, codéine, morphine et 6acétylmorphine dans le sang total. Toxicorama, 1996, 8 (2) : $17-22$

4. Smith R-A., Lewis D. Suicide by ingestion of T-61. Vet. Hum. Toxicol.. 1989, $31:$ 319-20.

5. Nicolas F., Rodineau P., Rouzioux J-M., Tack I., Chabac S., Meram D. Fulminant hepatic failure in poisoning due to ingestion of T-61, a veterinary euthanasia drug. Crit. Care Med. 1990, 18 : 573-75.

6. Buylaert W., Calle P., De Paepe P., Verstraete A., Samyn N., Vogelaers D., Vandelbulcke M., Belpaire F. Hepatotoxicity of $\mathrm{N}, \mathrm{N}$-dimethylformamide (DMF) in acute poisoning with the veterinary euthanasia drug T-61. Hum. Exp. Toxicol., 1996, 15, : 607-11.

7. Hanston P., Semaan C., Jouret J-C., Rahier J., Lauwerys R., Brunain J-P., Mahieu P. Intracardiac injection of T-61 a veterinary euthanasia drug. Clin. Toxicol. 1996, 34(2) : 235-39.
8. Giorgi M., Bertini S. Tanax® (T-61) : an overview. Pharmacol. Res. 2000, $41: 379-83$

9. Bertol E., Mari F., Bonelli A. Analytical toxicological studies in cases of suicide by injection of Tanax $\AA$, a veterinary euthanasia agent. J. Pharm. Biomed. Anal. 1983, $1: 373-7$.

10. Braselton E-V., Ray J-S, Slanker R-M, Rumler P-C. Determination of embutramide in mammalian tissues. Vet. Hum. Toxicol 1988, 30(6) : 536-9.

11. Huo J-Z., Bocxlaer J-V., Lambert W-L., De Leenheer AP. Determination of embutramide in biological matrices by gas chromatography with nitrogen-phosphorus detection. J. Chromatog B Biomed. Appl. 1994, 661 : 69-74.

12.Gaillard Y., Pépin G. Use of high-performance liquid chromatography with photodiode-array UV detection for the creation of a 600-compound library. Application to forensic toxicology. J. Chromatog. A 1997, 763 : 149-63

13. Hooijerink D., Schilt R., Brouwer B., Van Bennekom E. Determination of embutramide and pentobarbital in meat and bone by gas chromatography-mass spectrometry. Analyst. 1998, 123 : 2513-16.

14. Giorgi M., Bertini S., Soldani G., Giusiani M. Comparison of HPLC and GC-MS methods for determination of embutramide (a component of Tanax ${ }^{\circledR}$ or T-61) in biological specimens. J. Anal. Toxicol. 2001, 25 : 32327. 